\title{
Emergence of multitasking machining systems: applications and best selection practices
}

\author{
Manocher Djassemi \\ Industrial Technology Area of Orfalea College of Business, \\ California Polytechnic State University, San Luis Obispo, California, USA
}

\begin{abstract}
Purpose - Multitasking machining (MTM) systems have become increasingly sophisticated and expensive capital equipment. The lack of practical guidelines for selection of these machines can lead to significant undesirable machine attributes, application mismatch, and longer return on investment. The purpose of this paper is to provide an insight to numerous features and configurations of MTM systems and to present several application-based selection guidelines.

Design/methodology/approach - A taxonomy of MTM systems is developed based on the number of axes of motions, tooling and spindle systems. Practical guidelines for general and advance features are presented with special regard to multi-axis and multi-spindle features.

Findings - MTM systems are capable of meeting several production goals such as cycle time reduction, minimizing non-value added times and concurrent processing of multiple parts. However, they possess inherent programming challenges due to their complex configuration and simultaneous machining functions.

Research limitations/implications - The diversity of system configurations demand a decision support system, such as a rule-based expert system to capture the many variations of MTM systems. Originality/value - This paper should be useful to decision makers in industry or academia who are involved in selection of MTM systems.
\end{abstract}

Keywords Computer numerical control, Machine tools, Manufacturing systems

\section{Introduction}

Across all industrial markets and sectors the production challenges facing manufacturers revolve around cutting lead times, increasing throughput and getting products to market quicker. Other issues facing mass production shops include shorter runs, higher product mix, tighter tolerances, more complex geometries in tougher materials, and complete machining in a single handling (Koepfer, 2005). The introduction of new technologies and innovations has made a new generation of machine tools known as multitasking machining (MTM) systems more responsive to these challenges. MTM systems are computer numerical control (CNC) systems capable of performing a variety of operations with multiple tools and/or spindles in single setup. The advancement of computer technology and its application in manufacturing industry has transformed these systems from stand-alone production units to complex integrated workcells. These systems can contribute to significant savings in manufacturing operations due to their ability to complete complex workpieces with a multitude of features and geometries without the need for multiple setups and inter-machine part transfers. The tangible benefits of MTMs have drawn the attention of 
a large sector of the manufacturing industry including automobile, mold and die manufacturers, and aerospace companies as a means to gain substantial productivity, quality improvement and profit. However, MTM systems have become increasingly sophisticated and expensive capital equipment. Therefore, the selection of a system for the right application and at the right price is an important decision for a company and if it is not made properly it can lead to significant undesirable attributes with respect to user satisfaction and a long return-on-investment. As Hurco (2005) pointed out, the savings in setup time, increased part accuracies and reduction in inventories can be substantial if the machinery properly matches the application. This can be a challenging task for a potential buyer of multitasking systems who is faced with a wide range of machine configurations for which there are as yet, no industry-wide standards.

Much academic work on machine selection has focused on selection methodologies rather than providing practical guidelines to assist decision makers. In one of these studies Gopalakrishnan et al. (2004) discuss a two-phase decision support system for machine selection - a base machine selection and a detailed options selection. Factors such as cost and size of machine, floor space available, and the sizes of parts and fixtures were considered for the base machine selection. The detailed options selection phase considered aspects such as high speed, high accuracy, and complexity of cutting. Arslan et al. (2004) suggest a similar decision support system for machine tool selection by creating a sample database for a limited number of machine tools and applying a multi-criteria weighted average method to rank the alternatives. The database included information about machine type and size, spindle, tooling, work support, and axis of motion. The selection criteria consisted of precision, cost, reliability, safety, space, flexibility and productivity. The study of Selvaraj et al. (2006) points to appropriateness of MTM systems in the aerospace industry where the design of components changes frequently and there is a need for a machine that can adapt to each situation. The authors provide a guideline for selection of $b$-axis mill-turn machines. In study conducted by Chowdary (2007) an artificial neural network model is proposed as a cost effective solution for machining center selection problem. Several parameters were incorporated in the model including machine size, number of tools, tool changing time, spindle power and price. Lin and Yang (1996) propose an analytical hierarchy procedure for selection of conventional and CNC material removal machines. The procedure involved the evaluation of basic factors such as manufacturing lead time, labor cost and operation shifts.

These studies are typically limited to general machine tools or a subset of machine tools selection or non-material removal applications. However, the selection of modern MTM is more complicated compared to general machine tool selection because it involves the selection of numerous complex configurations and advance features including added axes of motions and spindles. There is little or no discussion about these configurations and features in published journal papers. This issue constitutes the framework of this study. Further, to assist the decision makers involved in MTM system selection a number of application-based selection guidelines are presented with emphasis on the advance features of the system.

\section{Framework of selection process}

Figure 1 shows a framework of factors involved in the decision-making process for a selection of a MTM system. Determination of production goals and available budget represent the starting point for MTM-selection process. Next, a decision is made to 
consider a basic two-five-axis machine or a more complex one with a higher number of axes, spindles and/or tool systems. At this stage a detailed analysis of needs and constraints determine the number of axes, spindle and type of tool systems that match the production goals. In final stage, general machine characteristics are determined to compliment the MTM-selection process. These factors are discussed in the following sections along with application recommendations.

\section{Production goals}

Before adopting a multitasking system a decision maker must have a clear understanding of the production goals for using such complex technology and major capital investment:

(1) Production flexibility. A key feature in MTM systems is flexibility, which takes various forms from machines that can do more because of multi-axis, multi-tool, and multi-spindle capabilities to those that can adapt to changes in product and market demand. MTM systems make low-volume work practical because there is less fixturing, equipment, and labor involved. This is particularly beneficial in situations with small batch sizes, a high variety of parts and highly specialized components where workpiece and tool changeover time might be substantial. Another measure of flexibility is the ability to use various stock geometries. With a multitasking system, a fabricator is no longer locked into using certain stock geometries, i.e. square or rectangular stock, because a part involves a lot of milling, or round stock because the part requires turning operations.

(2) Cycle time reduction. Most MTM systems are capable of processing multiple parts simultaneously using multiple spindles and/or axes of motion. Because of these attributes, once material is loaded on the machine, a completely finished workpiece is output allowing substantial reductions of in-process time.

(3) Lean manufacturing. Multitasking can contribute to a leaner operation by reducing non-value added times such as setup time as well as process simplification and streamlining:

- Setup reduction. Parts requiring multiple setups might be appropriate candidates for production on MTM systems. In addition to a reduction in the number of setups, setup time to total processing time can be used as an appropriate measure if the majority of time is spent on setup rather than production.

Figure 1.

The framework of factors

for selecting a

multitasking machining

systems

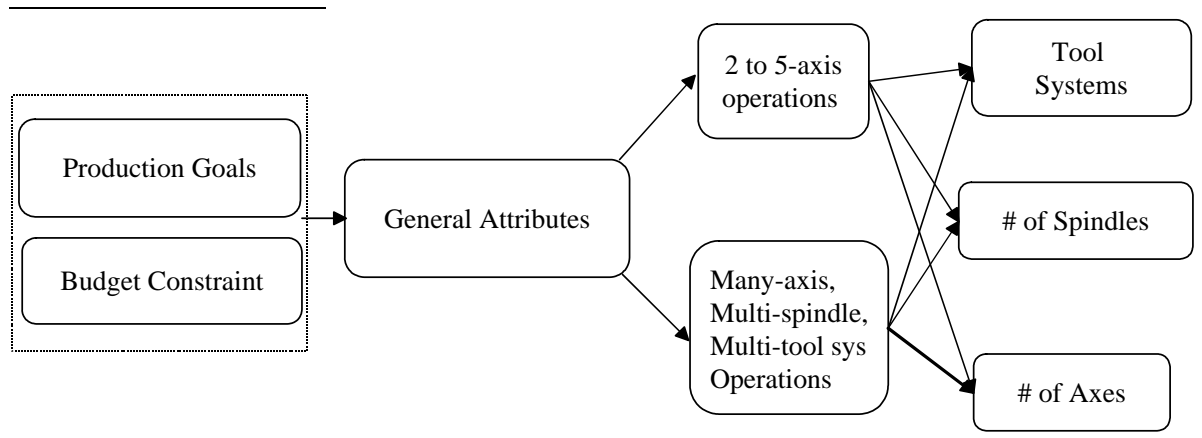


- Process simplification. It is also practical to cut complex parts entirely out of solid materials on a MTM system, instead of using a casting and machining sequence. Such process simplification not only can reduce manufacturing lead time but also results in higher quality parts compared to parts made by casting that may possess porosity. This approach may be more economical because tooling change in MTM system is potentially less expensive and time consuming than replacing casting molds or patterns if the part design is changed by the designer.

- Streamline machining. Most multitasking systems eliminate idle time by: enabling an axis to begin moving before another axis finishes moving; and allowing one tool to begin moving to position while the other is retracting.

(4) Quality control. MTM systems have the potential to improve part accuracy by not having to handle parts between operations, which eliminates the risk of stacked tolerances, reduces scrap rates and eliminates re-fixturing.

\section{General attributes}

The determination of general attributes of MTM systems including machine's body, way system, spindle drive system, etc. is not much different from conventional machine tools. A brief recommendation is provided for each feature:

- Machine's body. For the most part, machine tools utilize castings in their body because they exhibit good overall strength and vibration damping characteristics at a low cost. It is necessary for castings to have uniformly thick walls as variation in wall thickness can cause cooling and distortion problems. Along with castings many machines utilize weldments. Typically these are made of steel, cost less than castings and are stiffer and stronger when compared to castings of the same size and weight. Also, castings offer less damping characteristics. Weldments perform well at low speeds, but are more susceptible to vibration and chatter at high speeds, which can cause rough surface finishes (Cwik, 2006).

- Way system. The way system of a machine tool is one of the most important factors affecting characteristics such as part surface finish and overall accuracy. The way system has two main functions. The first function is to support the spindle and table, and the second is to guide the movement of the machine components. There are two primary types of way systems: box ways and linear guides. Of the two primary types linear guides provide faster positioning. This speed comes at a price as linear guides offer less vibration damping, less ability to withstand side thrust, and less ability to resist damage from crashes. Linear guides are often less expensive. Linear guides can be a good solution if the main materials to be machined are not difficult or require heavy rough cutting operations. Linear guides are also less suited for high-precision machining as tolerances and surface finish suffer from the reduced rigidity of the system. Box way systems offer less vibration, which in turn can lead to longer machine life. This also allows for the production of more accurate parts. The vibration damping characteristics also increase tool life and enable smoother surface finishes. Box ways do offer a better solution than linear guides for high accuracy and the machining of difficult materials. They also come with a high-initial price when compared to linear guides (Cwik, 2006). 
- Controls. The control of any machine is the brain which translates all the written code into precise movements and actions. The control interprets programmed code, sends commands to the machine to move its various components, monitors machine response, processes part programs and offers the ability to edit and fine tune existing programs. Some controls are more advanced and offer many added features. For facilities requiring the control of multiple machines networks can be developed between machines. This allows for increased monitoring, programming on separate machines, and file sharing between all machines and computers on the network, etc.

- Spindle drive system. The spindle is considered the heart of a MTM system. For consistent parts quality the spindle must maintain proper stiffness, rolling torque, spindle run-out, low-heat generation, and thermal stability. Machining different materials will affect these areas differently so there are many types of spindles, each for specific applications. There is not one best spindle for all applications. A spindle for high-speed aluminium machining most likely does not have the same material removal capability at low speeds as a spindle designed for low speed, high-torque cutting operations. Attention must also be paid to the type of spindle bearing due to its impact on stability and stiffness of the machine as a whole.

- Ergonomics. Ergonomics may seem like a low priority and is easy to ignore but the operator must have ready access to various areas of the machine to work effectively. For instance, the machine table should be within the reach of the control and located at an accessible height. Machines that provide easy access to parts and work pieces allow for faster setups and improved productivity. Along with the overall comfort and ease of operation, access to the various areas of the machine must not expose the operator to conditions that may be hazardous.

- High-speed machining. High-speed machines are defined by Kalpakjian and Schmid (2006) as machines that should provide spindle rotational speed between 2,000 to 6,000 feet/minutes for high speeds and 6,000-60,000 feet/minutes for very high-speed applications. The decision for adopting a high-speed MTM system is influenced by two major factors. First, the reduction of cycle time when cutting time accounts for a significant portion of overall machining time. Second, the faster dissipation of cutting heat through faster chip removal, which results in less thermal expansion and high-dimensional tolerances.

- Materials of workpiece. One of the basic requirements evaluated when determining performance needs is the type of materials to be machined. This will determine the levels of critical features such as spindle RPM, torque, and high-speed horsepower. If material removal operations mostly involve shaping soft materials like aluminium or brass the machines require higher speeds for finishing. On the other hand, if the operations mostly involve processing hard materials like steel or titanium the desired machines should encompass elements like low-speed torque, and rigidity to reduce the effects of side thrust.

\section{Multitasking attributes}

Most MTM systems contain five or more axes of motion, capable of utilizing any combination of $x, y, z, a, b$, and/or $c$-axes. This group of machines is commonly 
equipped with two or more tool systems and spindles and can operate in synchronous or asynchronous machining modes. An example of such machine is shown in Figure 2.

With the non-stop development of new machining methods and options, users must determine what type and configuration of MTM will best match their needs. Figure 3 shows a taxonomy of MTM systems that is constructed based on major structural and functional features including spindle orientation; number of axes, spindles and tool systems. While most MTMs can be identified as milling or turning type machines a hybrid MTM may

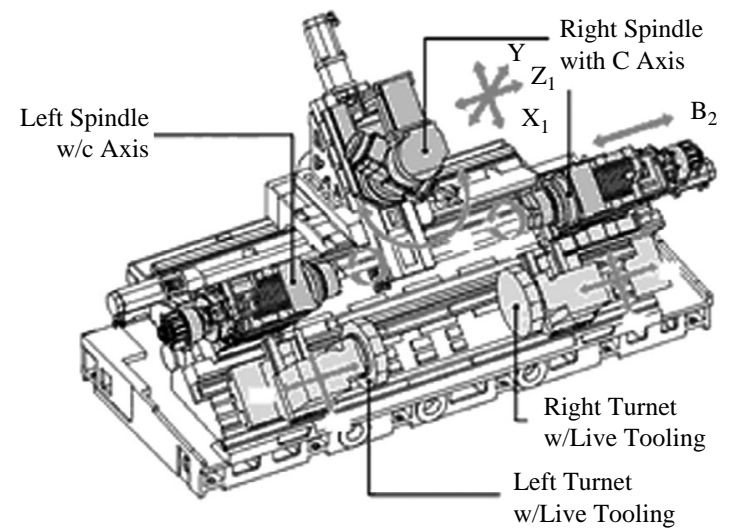

Figure 2.

An example of a multi-axis, multi-spindle and multi-turret system

Source: Courtesy of DP Technology




comprised of a wide range of functions such as turning, milling, contouring with the $c$-axis, off-center machining with the $y$-axis, milling of angled surfaces with the $b$-axis, heat treatment by laser, and grinding.

MTMs can be also subdivided into machines with vertical and horizontal spindle orientations. This structural feature which is inherited from conventional milling machines affects other machine features such as rigidity, chip excavation, machine size and fixturing capability.

The two basic configurations for cutting tool system in MTMs are carousel and chain tool magazines. All turning MTMs are equipped with turret-type tool system. Some hybrid MTMs offer combination of turret and special tool systems.

While machines with two-five axes have been in market for many years, the machine tool manufacturers have started adding more axes of motions and spindles to MTM systems. In the remainder of this paper, we focus on the impact of added axes and spindles on capabilities of MTMs and selection guidelines for various multi-axis/multi-spindle machine configurations.

\section{Multi-axis impact}

The number of axes of motion influences the performance of a MTM system in a number of ways. For instance, a five-axis machine can often equal the output of two three-axis machines and generate additional savings in inventory, floor space, and energy consumption (Neely, 2000):

- Part geometry. One of the major factors that influences the number of axes is the complexity of part geometry. Typically a multisided part or multisurface part requiring multiple setups is a good candidate for MTM. By machining complex shapes in a single setup, time is saved compared to performing the job in a series of setups. Five sides of a prismatic part, plus any combination of compound angles, complex sculptured parts, complex swept surfaces (e.g. turbine blades) can be machined in one setup.

- Work position maneuverability. Figure 4 shows the principal advantage of a five-axis machining system over a three-axis system and its ability to position the workpiece in several planes and/or a variety of angles to the spindle. With a three-axis system, spindle interference and long-reach machining can impose real problems when working with standard length tooling. For instance, certain geometrical features need end mill cutters as small as $1 / 8$ in. or $1 \mathrm{~mm}$ in diameter. Such slender tools are subject to deflection, which can result in machining inaccuracies and tool breakage. Moreover, this small cutter must be able to reach everywhere within a part cavity. That can create problems with three-axis machines where the spindle and toolholder are limited to a straight up and down movement and the girth of the toolholder prevents the tool from being able to reach such details as the radii, where the sidewalls meet the bottom of the pocket. This limitation may force the manufacturer to stop milling at some point and transfer the part to a secondary process such as an electro-discharge machine to complete the job. A five-axis MTM system overcomes this limitation by allowing non-perpendicular multi-axis tool movement (rotary axis $b$ or $c$ ) required for intricate features.

- Accuracy. For some applications the selection of a MTM system depends on how much movement or re-fixturing of the part can be eliminated rather than just 
Figure 4.

The effect of number of axes on work positioning

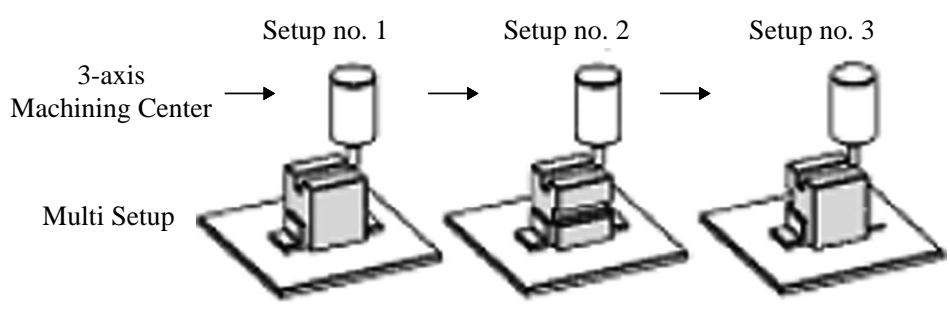

Setup no. 1

Table Rotation, Same Setup

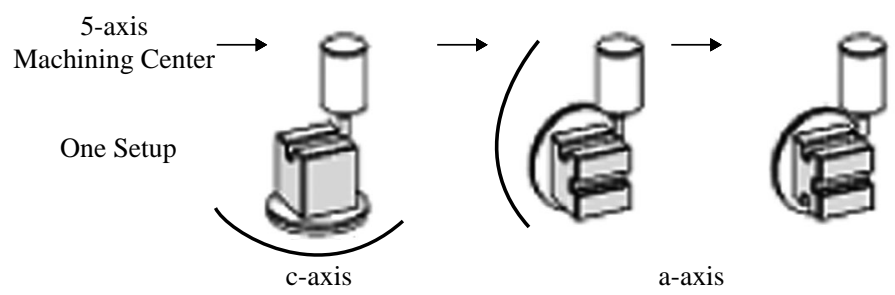

looking at complex geometry. This is because every time a part is moved, there is a potential for error. A piece of lint, dirt or debris can get behind the datum and affect the accuracy. In this case the objective of using a MTM system is to eliminate the potential for re-positioning error. Furthermore, with multiple setups, there is always the possibility of incorrect alignment each time the part is moved.

- High speed advantage. Conventional three-axis high-speed machining has limitations when milling deeper cores or cavities. The length of the cutters that have to be used with a three-axis system can result in chatter and poor surface quality. Five-axis machining enables shorter cutters to be used since the head can be lowered toward the job and the cutter-oriented toward the surface. As a result, higher cutting speeds can be achieved with no loss in accuracy.

- Many-axis advantage. When the number of axes in a MTM system goes beyond a common five-axis machine the ability to streamline the process by combining several operations can be enhanced significantly. Turning, milling, contouring with the $c$-axis, and off-center machining with the $y$-axis, are among many capabilities offered by such advanced hybrid MTM systems. One of the recent developments in design of multitasking systems is the addition of $y$-axis spindle and its associated rotary $b$-axis. A study by Koepfer (2006) indicates that 62 percent of turned pieces are potential candidates for turn/mill MTM systems. Of the 62, 25 percent are candidates for $y$-axis machining. In the following section we describe three configurations of recent many-axis MTM.

Configuration No. 1. Figure 5 shows a machine with two spindles, four tool turrets with live tool stations and 14 axes of motion including $x_{\mathrm{L} 1}, y_{\mathrm{L} 1}, z_{\mathrm{L} 1}, c_{\mathrm{L} 1} x_{\mathrm{L} 2}, y_{\mathrm{L} 2}, z_{\mathrm{L} 2}, x_{\mathrm{R} 1}$, $y_{\mathrm{R} 1}, z_{\mathrm{R} 1}, c_{\mathrm{R} 1} x_{\mathrm{R} 2}, y_{\mathrm{R} 2}, z_{\mathrm{R} 2}$ axes. By synchronizing the two spindles and cutting with four tools simultaneously material can be removed faster from the same part. This machine 
Figure 5.

A 14-axis MTM system with multiple spindles and turrets

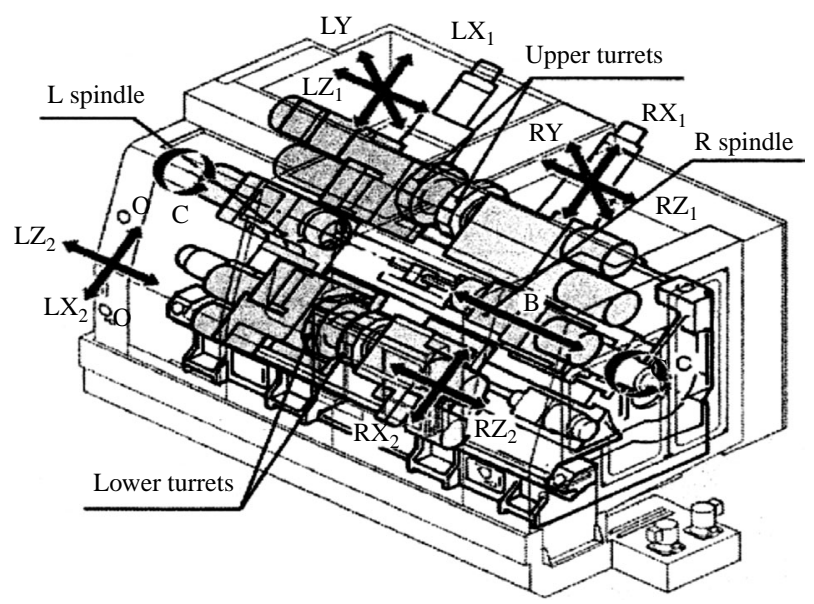

Source: Courtesy Namura-Tome

can also be used for shaping two identical parts by independent operation of spindles and turrets. Both configurations meet several production goals such as cycle time reduction and minimizing non-value added times.

Configuration No. 2. Figure 6 shows an 11-axis MTM system. This machine contains three tool systems and two spindles that are designed to function independently. This configuration is more suitable for machining the components that contain similar or dissimilar geometrical features on each end. These types of components would benefit from the functionality of a many-axis machine because they require numerous machining operations. They can also be made with minimal idle times required for tool changing, and minimal setup time for repositioning a workpiece. For instance, a sequence of machining operations may involve multi-diameter turning and threading, drilling holes, counterboring, slot drilling, milling of external flats, milling tiny spigots and ballnose milling all using a spindle in the right side of

Figure 6.

An 11-axis MTM system with axis, spindle and tool system designations

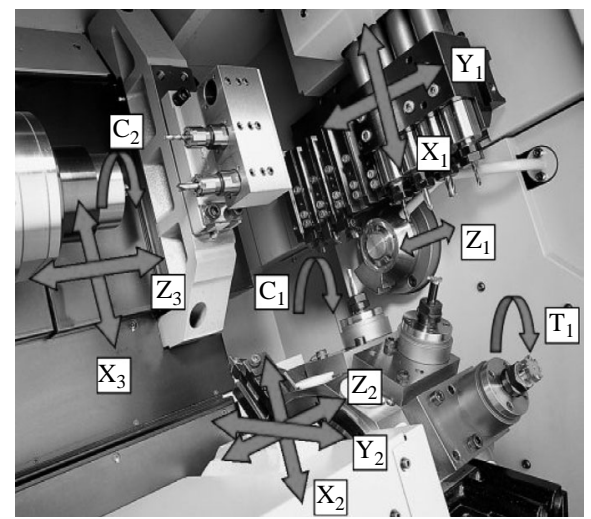

\begin{tabular}{|l|l|l|l|}
\hline $\begin{array}{c}\text { Axis } \\
\text { no. }\end{array}$ & Label & Spindle & Tool System \\
\hline 1 & $\mathrm{X}_{1}$ & $\mathrm{R}$ & vertical, turret \\
\hline 2 & $\mathrm{Y}_{1}$ & $\mathrm{R}$ & vertical, turret \\
\hline 3 & $\mathrm{Z}_{1}$ & $\mathrm{R}$ & vertical, turret \\
\hline 4 & $\mathrm{C}_{1}$ & $\mathrm{R}$ & vertical, turret \\
\hline 5 & $\mathrm{X}_{2}$ & $\mathrm{~L}, \mathrm{R}$ & vertical, turret \\
\hline 6 & $\mathrm{Y}_{2}$ & $\mathrm{~L}, \mathrm{R}$ & vertical, turret \\
\hline 7 & $\mathrm{Z}_{2}$ & $\mathrm{~L}, \mathrm{R}$ & vertical, turret \\
\hline 8 & $\mathrm{C}_{2}$ & $\mathrm{~L}$ & horizontal, turret \\
\hline 9 & $\mathrm{X}_{3}$ & $\mathrm{~L}$ & horizontal, turret \\
\hline 10 & $\mathrm{Z}_{3}$ & $\mathrm{~L}$ & horizontal, turret \\
\hline 11 & $\mathrm{~T}_{1}$ & $\mathrm{~L}$ & turret \\
\hline
\end{tabular}

Source: Courtesy of Citizen Machinery 
the machine. Simultaneously, in the left spindle, holes are drilled and tapped off-center and the part is drilled and bored.

As shown in Figure 1, a machine selection project involves considering many factors and constraints. One such factor is the size of the machine, which is a function of the available space and the range of part sizes. For instance, in the above two examples the 11-axis system occupies 50 per cent less floor space than the 14-axis system. However, the former machine can process much smaller parts than the latter one.

Configuration No. 3. Figure 7 shows a MTM system that is equipped with nine axes of motion, two turning spindles and two tool turrets. A distinctive feature of this machine configuration is the existence of a third spindle designated as $y$-axis and its associated rotary $b$-axis. This configuration allows shaping parts requiring multiple operations such as turning, drilling, boring, tapping and, particularly, milling of angled surfaces. In addition to combining many operations, this multitasking system will also ease the chip handling problem. That is, while the workpiece is spun in one direction, the y-axis mill spindle is spun in the opposite direction. The rotating milling cutter breaks the chips that would normally accumulate around the part (Destefani, 2003).

\section{Influence of multiple spindles}

Another factor to consider in selecting a MTM system is the number of spindles. Typically the spindles are operated either in synchronized fashion or independently. Dependent spindles can machine similar or different parts such as left- and right-hand parts. Independent spindles have separate drives that allow the system to alternately machine a workpiece:

- Production volume. Generally the output of a single spindle machine can be doubled or quadrupled by a twin- or four-spindle machine. Some MTM turning units even offer up to ten spindles that transform the CNC technology from a low to medium volume production system to a mass production system. Multi-spindle MTM systems are particularly suitable for high-volume runs and part families due to fast changeover.

Figure 7.

(a) A twin-spindle,

twin-turret plus $b$-axis

MTM system;

(b) a multi-faced part

suitable for $b$-axis

machining

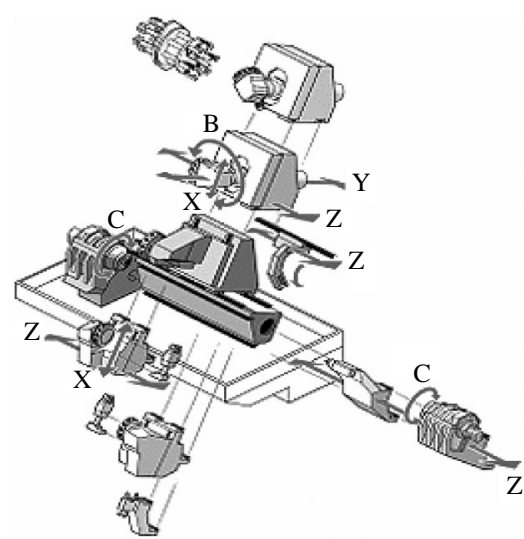

(a)

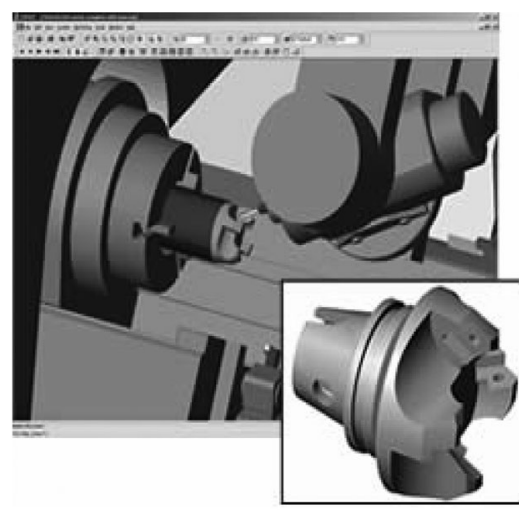

(b)

Sources: Courtesies of Index Corp. and Courtesy of DP Technology 
- Flexibility/utilization. Two other key issues that must be taken into consideration for using a multi-spindle MTM system are flexibility and utilization. With constantly changing orders and part types, having multiple spindles allows for concurrent processing of multiple part types. While, it may have a lower utilization rate per machine, the increased flexibility can justify the decision. Independent motor speeds and variable spindle speeds make the machining of different parts simultaneously on one machine as easy as producing a single workpiece.

- Space saving and part size. In the previous section, the capabilities of three MTM with multiple spindles and tool systems were discussed. Perhaps, a more obvious advantage of integrating multiple spindles and tool systems in one production unit is the efficient use of space in terms of footprint to work-space ratio. For instance, a two-spindle machine has the potential to deliver two times the productivity, within a footprint only slightly larger than that of a single spindle machine. However, as the number of spindles increases, the working envelope space does not increase by the same proportion. As a result, the size of parts that a machine can process is one of the key factors in determining the number of spindles for a MTM system.

- Maintenance cost savings. A less obvious advantage is lower maintenance cost. That is, multiple spindles in one machine utilize a shared common fluid system and other machine resources that would otherwise require separate maintenance on separate machine tools (Zelinski, 2006).

\section{Cost considerations}

Cost is more often than not the driving factor in the selection of a production system. With this being said MTM systems are expensive. The higher the number of axes, spindles, and tool turrets the higher the price, so a company must thoroughly analyze their goals and needs before starting the search for addition of a MTM system to their machine arsenal. However, to get the most from their investment, companies have to match their needs to the machine's characteristics, features, and options. The key issue in decision making for purchasing a MTM system is not to bypass features like $b$-axis capability, extra spindles or turrets, just because they add extra costs. These features provide enormous flexibility for a company for changing the design of a product in response to market demands at a cost which will be insignificant over the life of the machine.

\section{Challenging issues}

In spite of the numerous advantages discussed in this paper, a company that plans to add a MTM system to its production line must be aware of some challenging issues involved in using these machines. For instance, MTM systems possess inherent programming challenges due to their complex configuration, simultaneous machining, and non-perpendicular multi-axis tool movement (Mathews and Stoveland, 2006). The number of axes and tool turrets in MTM systems can be intimidating for some users. First time users might be over-cautious due to their "fear factor." This is understandable considering the fact that several axes and tool systems are operational within a fairly limited work envelope and that the workpiece and tooling pass within very close proximity to each other. To overcome this challenge, several computer-aided manufacturing software packages are available that provide visual verification of machine motions for error-checking and collision detection (UGS, 2007). 
A closely related issue is the reliability of MTM systems. Integration of multiple functions in a single production unit while providing the opportunity for higher productivity, may increase the sensitivity to machine reliability, especially, in the case of machine breakdown. One approach to deal with this issue is selection of a machine with independent spindle and/or axis drive systems.

Another challenging issue facing MTM systems is post-processing. Most MTMs can require multiple post-processors for various combinations of milling and turning on various turrets and spindles (Figure 7). A remedy for this problem is available in the form of a control software that automates the post-processor selection based on the sequence of operations on upper or lower turrets and left or right spindles (UGS, 2007).

\section{Summary and further research}

MTM systems provide enormous capability for manufacturing a wide range of products at a cost which will be insignificant over the life of the system. In contrast to previous studies (Gopalakrishnan et al., 2004; Selvaraj et al., 2006) in which only basic features of $\mathrm{CNC}$ machining centers have been discussed, in this study we presented a review of basic as well as advance features of MTM systems along with a guideline for selection of these features.

In summary, the most important features and applications of MTM systems as discussed in our study are:

(1) Five-axis/single spindle/single tool system:

- higher accuracy due to elimination of part repositioning;

- shaping at various angles due to work position maneuverability; and

- higher machining speeds due to use of shorter cutting tools.

(2) Five to twenty axes, two-ten spindles, two to four systems (in addition to above features):

- simultaneous shaping of two or more identical parts;

- simultaneous shaping of non-identical parts;

- milling angled surfaces on cylindrical shape parts; and

- multiple operations: milling, turning, grinding, etc.

While these systems are capable of meeting several production goals such as cycle time reduction, minimizing non-value added times and concurrent processing of multiple parts, they possess inherent programming and reliability challenges due to their complex configuration and simultaneous machining functions.

Closely related issues are the tooling and fixturing aspects of MTM systems, which were not included in this study. Although, it typically represents a very small percentage of total system cost, tooling can have a disproportionate impact on throughput and total parts cost (Destefani, 2003). In recent years, machine tool builders and cutting tool suppliers have paid more attention to new tool designs that can take better advantage of the MTM unique capabilities. A study to reflect the results of these efforts and explore the tooling needs of MTM systems would compliment the guidelines presented in this paper.

While in this study we discussed a number of common configurations of multitasking systems, a decision support system, such as a rule-based expert system, 
is needed to capture the many variations of these configurations. Such expert systems can be expanded by incorporating a database of manufacturers, models, accessories, and associated costs.

\section{References}

Arslan, M., Catay, B. and Budak, E. (2004), "A decision support system for machine tool selection”, Journal of Manufacturing Technology Management, Vol. 15 No. 1, pp. 101-9.

Chowdary, B. (2007), "Back-propagation artificial neutral network approach for machining center selection", Journal of Manufacturing Technology Management, Vol. 18 No. 3, pp. 315-32.

Cwik, G. (2006), “One way to select a vertical machining center”, Modern Machine Shop, available at: www.mmsonline.com/articles/079804.html

Destefani, J. (2003), "New tooling designs can help you maximize multitasking productivity", Manufacturing Engineering, Vol. 130 No. 4.

Gopalakrishnan, B., Yoshii, T. and Dappili, S.M. (2004), "Decision support system for machining center selection", Journal of Manufacturing Technology Management, Vol. 15 No. 2, pp. 144-54.

Hurco, R.A. (2005), “A pivotal solution”, Fabrication and Metalworking Magazine, January.

Kalpakjian, S. and Schmid, S.R. (2006), Manufacturing Engineering and Technology, 5th ed., Prentice-Hall, Englewood Cliffs, NJ, p. 778.

Koepfer, C. (2005), "Rethinking the multi-spindle", Production Machining, available at: www. productionmachining.com/articles/050501.html

Koepfer, C. (2006), "Why y-axis for turn/mill machines", Modern Machine Shop, available at: www.mmsonline.com/articles/099503.html

Lin, Z. and Yang, C. (1996), "Evaluation of machine selection by the AHP method", Journal of Materials Processing Technology, Vol. 57, pp. 253-8.

Mathews, C. and Stoveland, E. (2006), "A-axis turn/mills have their place”, available at: www. mmsonline.com/articles/090302.html (accessed December 2006).

Neely, E.C. (2000), "Five-axis is worth it", Manufacturing Engineering, November, p. 39.

Selvaraj, P., Thirumal, E. and Radhakrishnan, P. (2006), "Multitasking machines: a new approach to increase the productivity of aircraft components manufacture", International Journal of Computers in Technology, Vol. 27 No. 1, pp. 24-30.

UGS (2007), "The challenges of programming multi-function machines", White Paper, available at: www.ugs.com (accessed January 2007).

Zelinski, P. (2006), “When two spindles are better than one”, Modern Machine Shop, available at: www.mmsonline.com/articles/079804.html (accessed December 2006). 